\title{
NEUROPATHIC KERATITIS A RESULT OF FOCAL INFECTION.
}

\section{J. W. Charles, M.D., F.A.C.S.}

\section{ST. LOUIS, MO.}

The views here advanced are that dendritic keratitis and also neuropathic keratitis are caused by lesions of the terminal divisions of the corneal nerves. Illustrations are given showing the form of the nerve terminal branches in the cornea of the dog. A case of neuropathic keratitis is reported, which rapidly cleared up after treatment of subacute ethmoiditis on the side of the affected eye. Read before the St. Louis Ophthalmic Society. March 24, 1922. See p. 736.

In the April, 1904, number of Dr. Alt's American Journal of OphthalMoLocy, I advanced the theory that rendritic keratitis was a terminal nerve lesion; because of the accompanying corneal anesthesia, the shape of the lesion, especially at the beginning, the advancement of the ulcer by new infil-

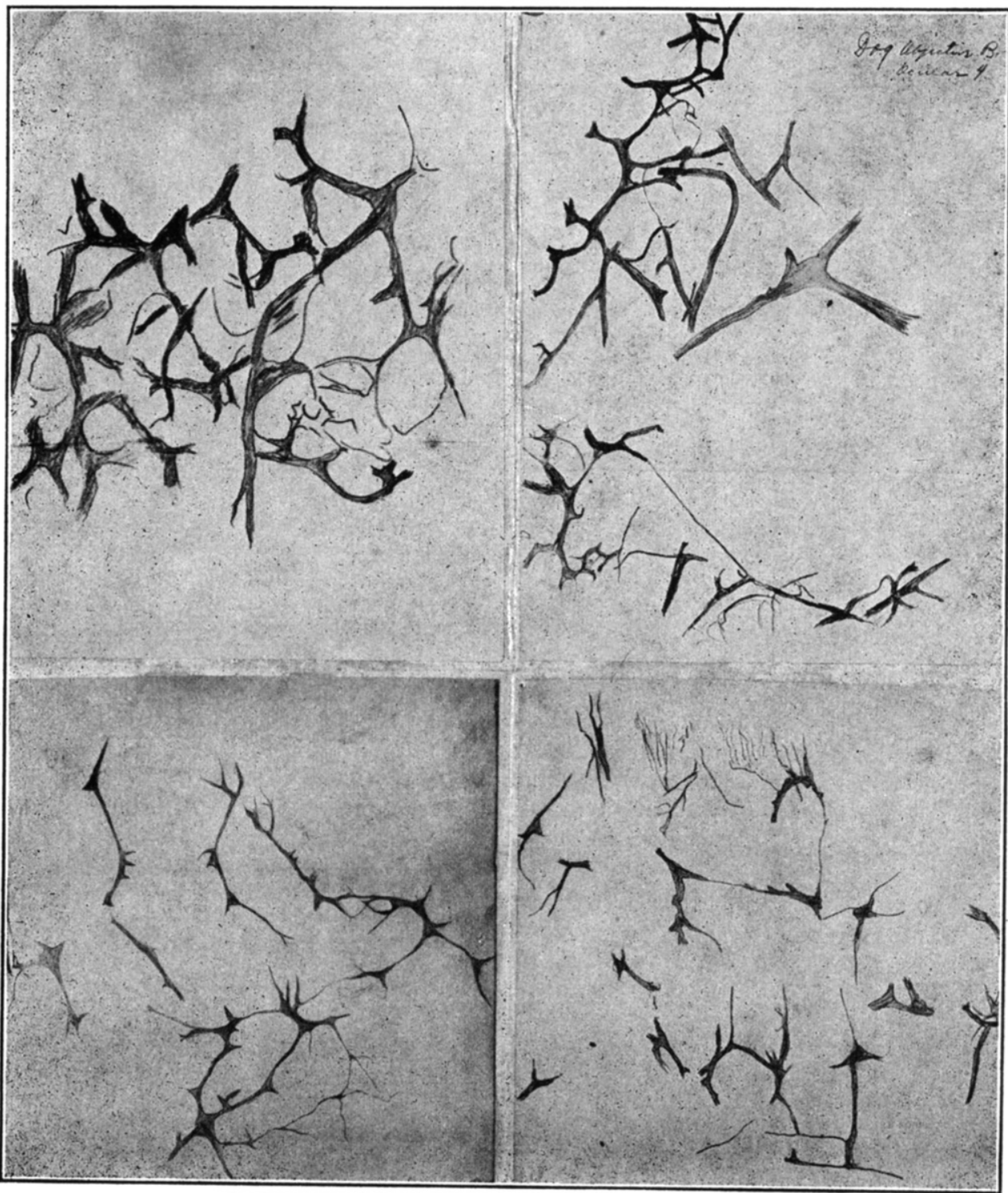

Fig. 1. Photographs of Drawings of the Subepithelial Nerve Plexus in a Dog's Cornea Made with Zeiss' drawing apparatus Ohjective $\mathbf{B}$, ocular 4. 
trations as if along the branching of a nerve, and also the extreme chronicity of the disease and the fact that it is seen only during or after those diseases which, when they affect the nervous system, affect the terminal nerves rather than the central nervous system, e. g., malaria, grippe, pneumonia. At that time I reported the case of a man with typical dendritic keratitis who had had grippe; and I contended, that

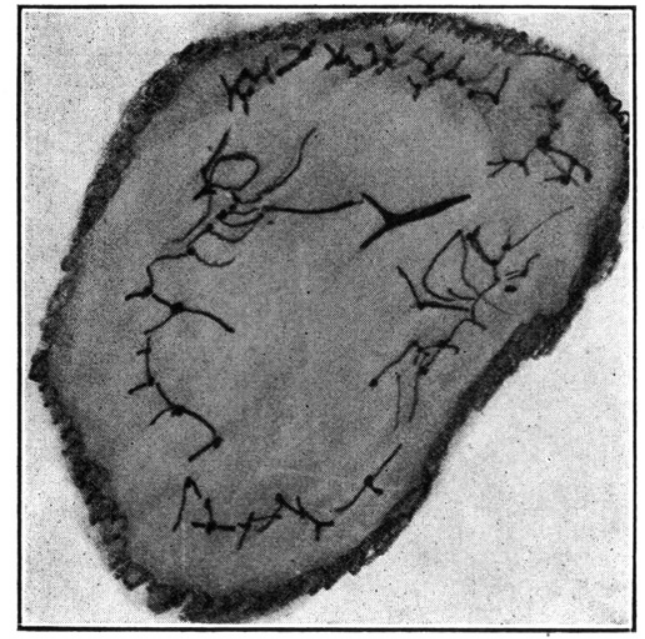

Fig. ?
In order to substantiate the theory that such terminal lesions are possible from focal infections, one needs only to point to the various "neuralgias," without other apparent complication, which have been relieved by suitable treatment of the offending focus.

Dr. W. A. Shoemake: first suggested the idea of such a cause for a neuropathic keratitis, in a case which he accorded me the privilege of seeing sev-

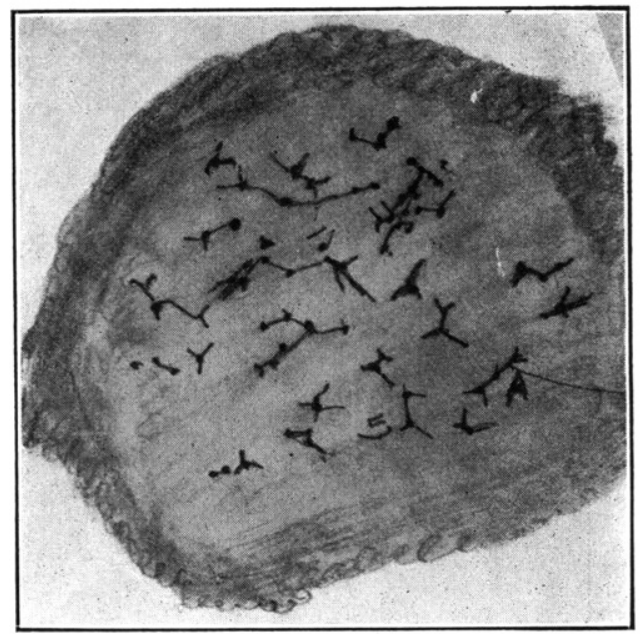

Fig. 3

Fig. 2 and Fig. 3. Demonstrate the Triangular Nodes and Nerve Bundles as Visible to the Unaided Eye. Sections $2 \mathrm{~mm}$. in diameter, magnified 32 diameters. Actual size of $\mathrm{A}$ in Fig. $3,1 / 7 \mathrm{~mm}$.

while herpes corneæ is vesicular, the typical malarial keratitis is papular and leaves a much more obstinate lesion than results from herpes.

With the Zeiss drawing apparatus for the microscope, I also made drawings of the subepithelial nerve plexus of a dog's cornea, and found that I could reproduce a very convincing combination of appearances of dendritic keratitis by substituting dots and fine lines for the triangular branching nodes and bundles of nerve fibrils, as will be noted in these crude drawings, - not proof but to me somewhat significant. I then proved that if such bundles of fibrils become opaque, or their channels filled with leucocytes, either could be visible to the unaided eye, by comparing them with the finest gossamer, which I was able to quarter mentally under the microscope. eral months ago, and which he will later describe.

My own patient, a 22 year old girl, of a rather neurotic type, came to me October 12, 1921, with the history that she had had influenza two weeks before, followed by chills and fever five or six times. Dr. J. W. Shaw gave the patient quinine, 3 grains, every three hours for seventy-two hours, and then t.i.d., since which course she has had no chills or fever.

The right eye first began to water and become red six days before I saw her.

Present Condition. There is a small branching ulcer of the cornea, which is accompanied by great photophobia, lacrimation and ciliary injection. On October 21st, the ninth day of treatment, V. R. 23/48 under atropin, em- 
metropic. V. L. 23/24, also emmetropic. Treatment consisted in the usual remedies, including iodin and finally mercurochrome, atropin several times a day with holocain, and bandage.

On November 3rd, she was sent to her dentist, who placed her teeth in good order, after having found a right lower third molar badly decayed.

Having exhausted all other methods known to me, on November 16th I referred the patient to a rhinologist, Dr. Roy Scholz, who found a mild suppurative subacute ethmoiditis on the right side, which responded beautifully to palliative treatment. From the time he began to treat the patient daily, by shrinking the sinuses, and by

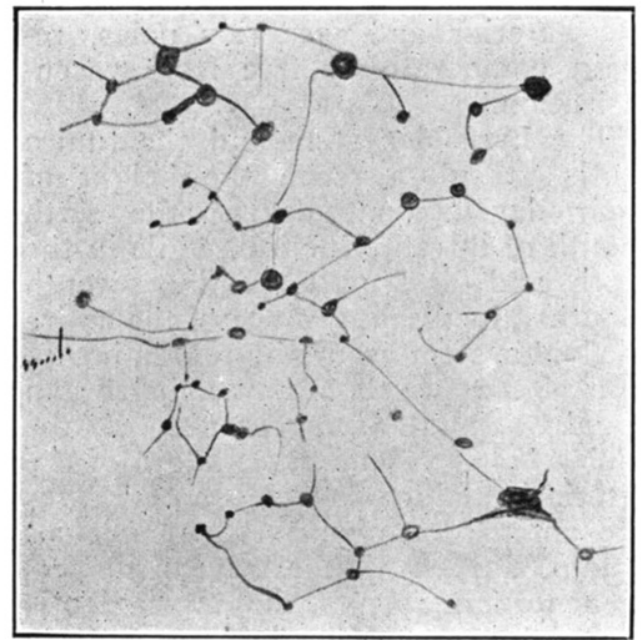

Fig. 4 applying mild astringent antiseptics, the eye began to improve,-- in fact two days after she was referred the ulcer had healed, altho the ciliary injection lasted some time longer.

Dr. Scholz's letter to me in February states, "When I last saw her I was unable to extract pus from the ethmoids; the mucosa of ihe entire nose was free from congestion and the patient was entirely free from symptoms."

On December 13 th there was only a faint scar remaining, very little photophobia and the vision was: R. 23/24, Em. ' L. 23/19, Em.

The patient was last scen December 23rd, and the eye was free from symptoms or signs of disease.

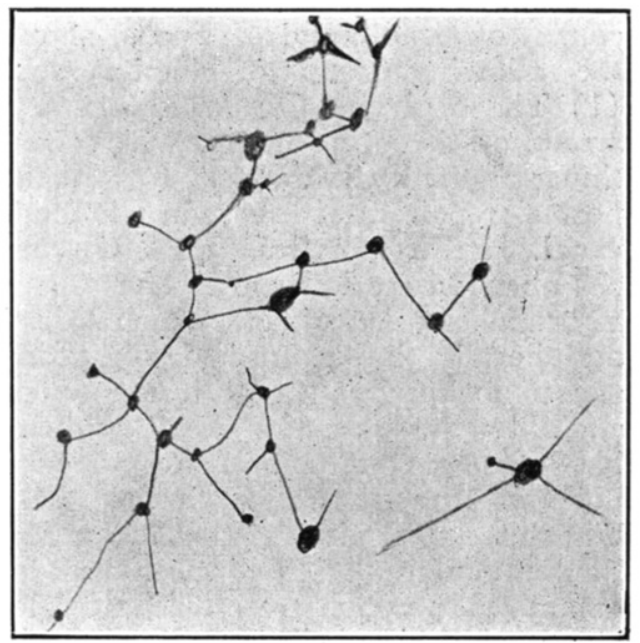

Fig. 5

Fig. 4 and Fig. 5. Dots drawn exactly width of Triangular Branching Nodes which will be Visible to the unaided eye if opaque and under proper illumination. Average width of Fiber Bundles 11.4 micra. Author's unaided eye quarters a gossamer thread 22 micra in diameter. 